Katharina König

Beisitzerin - zuständig für Mitglieder in Ausbildung

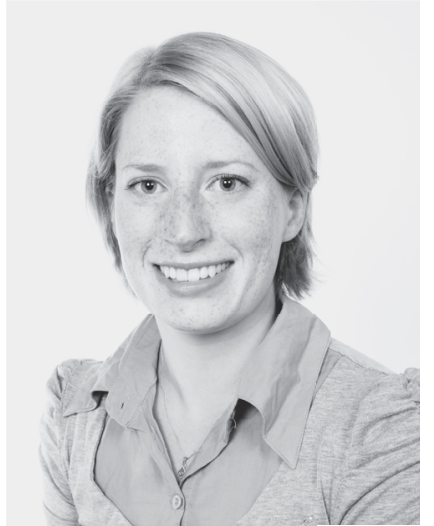

Ich bin 1986 in Berlin geboren und auch, wenn mich nun etwa 800 Kilometer von meiner Heimatstadt trennen, komme ich immer wieder gerne zurück und staune, wie sich diese Stadt ständig neu erfindet. Ich freue mich darauf, als Beisitzerin im BuVo nun feste Termine in der Hauptstadt zu haben.

Nach meinem Grundstudium an der FU bin ich an die

Freiburger Uni gewechselt, an der ich inzwischen mein Schwerpunktstudium im Bereich Rechtsgeschichte und Rechtsvergleichung beendet habe und hoffentlich im nächsten Jahr mit dem 1. Examen das Studium abschließe.
Ich habe während meiner Schulzeit an einem altsprachlichhumanistischen Gymnasium ein halbes Jahr lang in einem Internat in Irland gelebt und im Rahmen eines Praktikums das Londoner Leben kennen gelernt. In der spannenden Zeit des Bundestagswahlkampfs 2005 konnte ich im Rahmen eines Praktikums beim SPD-Landesverband Berlin einen ersten Einblick in die Politik gewinnen. Später habe ich im BMJ die Ministerialarbeit kennengelernt. In Freiburg bin ich am Institut für Ausländisches und Internationales Privatrecht beschäftigt. Zuvor habe ich in Berlin Medien-, Kunst- und Urheberrecht für mich entdeckt und eine tolle Zeit in der Kanzlei von Peter Raue verbracht. Dort traf ich den gegnerischen Anwalt Christian Schertz. In seiner Kanzlei war ich als Aushilfe tätig und konnte so die vielen spannenden Seiten des Anwaltsberufs kennenlernen.

djb-Mitglied bin ich seit diesem Jahr. Im Schultheater spielte ich die antike Vorkämpferin des Rechts Antigone und habe den Kern der Figur verinnerlicht: Nach ihrem Vorbild möchte ich mich mutig für das Recht einsetzen.

\title{
Der djb gratuliert
}

Prof. Dr. Dr. Juliane Kokott zum Antritt ihrer zweiten sechsjährigen Amtszeit als deutsche Generalanwältin am Europäischen Gerichtshof am 7. Oktober 2009.

Prof. Dr. Beate Rudolf zur Wahl als Direktorin des Deutschen Instituts für Menschenrechte in Berlin. Sie wird ihr Amt am 1. Januar 2010 antreten. Sie ist seit 2003 Juniorprofessorin für Öffentliches Recht und Gleichstellungsrecht an der Freien Universität Berlin, seit 2006 zugleich Leiterin des Sonderforschungsprojekts „Völkerrechtliche Standards für Governance in schwachen und zerfallenden Staaten “ an der Freien Universität.

\section{Trauer um Dr. Hanna Beate Schöpp-Schilling}

Zusammen mit Frauenrechtlerinnen in Deutschland und weltweit trauert der djb um sein Ehrenmitglied Dr. Hanna Beate Schöpp-Schilling. Wir verlieren einen wunderbaren Menschen, eine leidenschaftliche Streiterin für die Rechte der Frau und ein engagiertes Mitglied der djb-Kommission „Öffentliches Recht, Europa- und Völkerrecht“.

Dr. Beate Schöpp-Schilling war geprägt durch die familiären Erfahrungen im Zweiten Weltkrieg, die Not der Nachkriegszeit und den Wiederaufbau einer Existenz aus dem Nichts. Die Aussöhnung mit dem jüdischen Volk und mit Deutschlands östlichen Nachbarn war ihr bis zum Schluss wichtig. Wesentlich für ihre Entwicklung waren die USA - zunächst ein High School-Jahr, später mehrere Studienaufenthalte. In diesem Herbst wird das 50jährige Jubiläum des High
School-Diploms gefeiert, und sie hatte sich so darauf gefreut, daran teilzunehmen ...

In den USA war es zunächst die Offenheit des Denkens, die sie beeindruckte. Hinzu kamen die Erkenntnisse aus der Befassung mit Frauenstudien, die sie zur überzeugten Feministin machten und die ihr weiteres Berufsleben prägen sollten. "Scratch a woman and out comes a feminist", so hat sie selbst es charakterisiert. Aus den USA brachte sie die Idee einer Sommeruniversität für Frauenstudien mit und sie verwirklichte als erste diese Idee in Deutschland. Ein fortdauerndes Zeichen ihres Wirkens ist die Existenz einer Zentraleinrichtung für Frauen- und Geschlechterstudien an der Freien Universität Berlin, deren Schaffung maßgeblich auf Dr. Beate SchöppSchilling zurückgeht. 
Wohl der Hauptgrund, nicht die Wissenschaftslaufbahn einzuschlagen, war die in den USA gewonnene Erkenntnis, dass Frauenforschung und Frauenpolitik verbunden werden müssen. Den entscheidenden Schritt in die Frauenpolitik tat sie 1987, als sie die Geschäftsführung des Aspen-Instituts in Berlin aufgab, um als Parteilose in das Bundesfrauenministerium unter Rita Süssmuth einzutreten und dort Abteilungsleiterin der neu gegründeten Abteilung Frauenpolitik zu werden. Sie selbst hat ihren Einsatz nach der Wiedervereinigung als ihre wesentlichste Leistung angesehen: etwa die Schaffung kommunaler Gleichstellungsbeauftragter, die institutionelle Verankerung von Gleichstellung und Frauenförderung oder die Finanzierung von Frauenhäusern in den neuen Ländern.

Das Jahr 1989 brachte ihre Wahl in den UN-Ausschuss zur Beseitigung der Diskriminierung der Frau, dem sie zwanzig Jahre lang angehören sollte. Wie wohl kein anderes Mitglied des CEDAW-Ausschusses hat sie dessen Arbeit geprägt. Hier konnte sie ihre

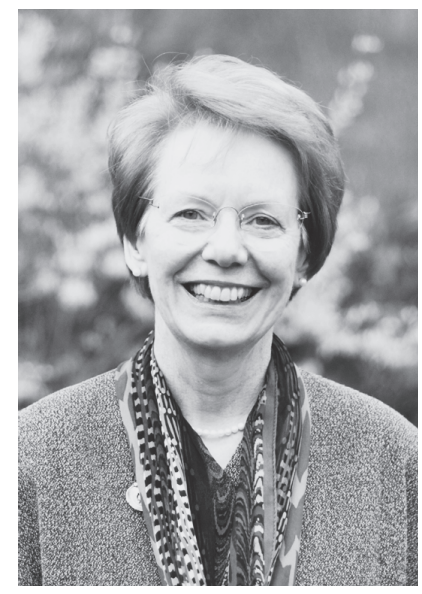
Stärken einbringen: die Fähigkeit zu scharf-

Wer mit Beate zusammengearbeitet hat, weiß: Ihre hervorstechenden Eigenschaften waren ihre produktive Ungeduld und ihre Hartnäckigkeit. Sie spornte immer wieder zu konsequentem Fortführen begonnener Aktivitäten und zu neuen Initiativen an. Stillstand gab es bei ihr nicht. Wir in der djbKommission profitierten von ihren Erfahrungen mit Frauenpolitik in Deutschland und weltweit, von ihren praktischen Anregungen und strategischen Überlegungen. Viele Frauen verdanken ihr freundschaftlichen Rat, Ansporn und Förderung.

Beate verlangte viel von anderen, mehr aber noch von sich selbst. Zusätzlich zu ihrer CEDAW-Arbeit schulte sie weltweit Vertreterinnen und Vertreter von Nichtregierungsorganisationen und Regierungen sowie Abgeordnete im Bereich Menschenrechte und Gleichstellung von Frauen. Darüber hinaus war sie wissenschaftlich ungeheuer produktiv. Außerdem engagierte sie sich in zahlreichen Stiftungen ehrenamtlich und betätigte sich in ihrem Heimatort Wohltorf kommunalpolitisch.

Wohlverdient waren deshalb die Ehrunsinniger politischer und juristischer Analyse, gepaart mit gesundem Pragmatismus, den Erfahrungen aus innerstaatlicher Frauenpolitik und eine schier unermüdliche Arbeitskraft. Dass der Ausschuss heute kohärent arbeitet, dass CEDAW als Rechtsinstrument wahrgenommen wird, dass die in der Konvention garantierten Frauenrechte als Menschenrechte verstanden werden - all das geht in einem großen Maße auf Dr. Beate Schöpp-Schilling zurück. Sie hatte in der Zeit ihres Wirkens verschiedene Positionen im Ausschuss inne, darunter zweimal den Vizevorsitz des Ausschusses (1999-2000, 20052006) und die Leitung der Ständigen Arbeitsgruppe zum Beschwerdeverfahren (2000-2004). gen, die sie in den letzten beiden Jahren erfuhr: zunächst die Ehrenmitgliedschaft im Deutschen Juristinnenbund, dann die Verleihung des Margherita-von-Brentano-Preises der Freien Universität Berlin (vgl. djbZ 2009/2) und schließlich im Mai die Verleihung des Bundesverdienstkreuzes Erster Klasse.

Viel zu früh wurde Beate aus unserer Mitte gerissen. Ihr Lebenswerk ist ihr Vermächtnis und unsere Verpflichtung. Uns bleibt die Dankbarkeit für ihre Leistungen und ihre Freundschaft.

Prof. Dr. Beate Rudolf

Mitglied der Kommission Öffentliches Recht, Europa- und Völkerrecht des djb, Freie Universität Berlin

\section{Geburtstage}

(August bis Oktober 2009)

\section{Jahre}

\author{
- Adelheid Harthun-Kindl \\ Präsidentin des LSG a.D. \\ Berlin
}

Dr. Utta Kaiser-Plessow Richterin am FG i.R. Köln

\section{Jahre}
- Dr. Melitta Büchner-Schöpf Ministerialdirigentin a.D. Unternehmerin Berlin

- Dr. Barbara Höynck-Lüthgen Botschaftsrätin i.R. Wachtberg-Villip

- Ursula Lüling Rechtsanwältin Stuttgart

\section{Jahre}

\author{
- Edith Endrös-Baum \\ Rechtsanwältin \\ Stockdorf
}

